Pacific Journal of Mathematics

SEPARATE CONTINUITY AND JOINT CONTINUITY 


\section{SEPARATE CONTINUITY AND JOINT CONTINUITY}

\section{NAMIOKA}

The main theorem is somewhat stronger than the following statement: Let $X$ be either a locally compact Hausdorff space of a complete metric space, let $Y$ be a compact Hausdorff space and let $Z$ be a metric space. If a map $f: X \times Y \rightarrow Z$ is separately continuous, then there is a dense $G_{\delta^{-}}$set $A$ in $X$ such that $f$ is jointly continuous at each point of $A \times Y$. This theorem has consequences such as Ellis' theorem on separately continuous actions of locally compact groups on locally compact spaces and the existence of denting points on weakly compact convex subsets of locally convex metrizable linear topological spaces.

o. Introduction. The consideration of separate continuity vis$\grave{a}$-vis joint continuity goes back, at least, to Baire (1899), whose work is the prototype of all the subsequent investigations on this subject by many mathematicians. The general problem is $(P)$ : find conditions on topological spaces $X, Y$, and $Z$ so that each separately continuous function $f: X \times Y \rightarrow Z$ (i.e., function continuous in each variable while the other variable is fixed) is jointly continuous at points of a "substantial" (in some topological sense) subset of $X \times Y$. As far as we know, all the available answers to this question require that either $X$ or $Y$ be metrizable or satisfy some sort of countability condition (e.g. [4, §5 Problem 23] and [9, Theorem 3]). This requirement severely restricts their applications. For instance, the proof of Ellis' theorem [7, Theorem 1] is fairly complicated because a step by step reduction of the general case to the metrizable case is involved. This also explains why the theorems on weak-compact sets in [18] require the separability condition.

However, the recent result of Troyanski [21] on renorming a Banach space that is generated by a weak-compact set enables one to drop the separability condition from many theorems on weak-compact subsets of Banach spaces. Indeed, it is relatively simple to deduce the following answer to problem $(P)$ from Troyanski's renorming theorem: Let $X$ and $Y$ be compact Hausdorff spaces and let $f$ be a bounded separately continuous real-valued function on $X \times Y$. Then there are dense $G_{\hat{o}}$-sets $A$ and $B$ of $X$ and $Y$ respectively such that $f$ is jointly continuous at each point of $A \times Y \cup X \times B$. This indicates that some good answers to problem $(P)$ have been overlooked in the past. 
The purpose of the present paper is to give an answer (Theorem 1.2) to problem $(P)$ that generalizes the one which follows from Troyanski's theorem, and to describe some applications of Theorem 1.2 .

Section 1 begins with a discussion of a class of topological spaces which is used in the statement of the basic theorem (Theorem 1.2). The statement and the proof of this theorem occupy the rest of the section. The proof employs, in addition to the usual category argument, an argument based on a double sequence, which is reminiscent of the poof of Eberlein's theorem.

In $\S 2$, the basic theorem is recast as theorems on function spaces. This formulations is sometimes useful.

In $\S 3$, we prove a generalization of Ellis' theorem [7, Theorem 1] and a theorem of Corson and Glicksberg [6, Theorem 1] not, however, in the generality as stated in [6] (cf. Remark 3.4). Both theorems follow immediately from the basic theorem.

In $\S 4$, the method of [18] is combined with the basic theorem to prove Troyanski's theorems on weak-compact sets [e.g. a weakcompact convex subset of a Banach space is the closed convex hull of its "denting points" (see the definition in §4)]. Since our method is linear topological, metrizable locally convex linear topological spaces are more natural settings for these theorems. Finally we discuss weak*-compact subsets of a dual Banach space that is generated by a weak-compact set. Problem 16 of Lindenstrauss [17] is settled affirmatively.

In most cases, the notation and terminology are those of Kelley [13] and Kelley, Namioka et al. [14].

Finally, we wish to express our gratitude to the following friends and colleagues whose suggestions and encouragements resulted in a substantial improvement of the final version over the preliminary draft: Professor H. Corson, Professor J. Dugundji, Professor E. Michael, Dr. R. Olson, and Professor R. Phelps.

1. The basic theorem. In order to state the basic theorem in a unified manner, we shall use a convenient class of topological spaces that includes locally (countably) compact spaces and complete metric spaces. Let $\mathscr{A}$ be an open covering of a topological space $X$. Then a subset $S$ of $X$ is said to be $\mathscr{A}$-small if $S$ is contained in a member of $\mathscr{A}$. A topological space $X$ is said to be strongly countably complete [11], if there is a sequence $\left\{\mathscr{A}_{i}: i=1,2, \cdots\right\}$ of open converings of $X$ such that a sequence $\left\{F_{i}\right\}$ of closed subsets of $X$ has nonempty intersection provided that $F_{i} \supset F_{i+1}$ for all $i$ and each $F_{i}$ is $\mathscr{A}_{i}$-small. A locally countably compact space is strongly countably complete. Clearly a closed subspace of a strongly countably complete space is 
again strongly countably complete, but the product of two strongly countably complete spaces may not be strongly countably complete [11]. There is a class of strongly countably complete spaces with more pleasant permanence properties. A Tychonoff space (i.e., a completely regular $T_{1}$-space) $X$ is said to be Cech-complete ("complete in the sence of Čech" in [8]) if and only if $X$ is a $G_{\delta}$ in its Stone-Čech compactification, and this is the case if and only if $X$ is a $G_{o}$ in some comppactification of $X$ [8, Theorem 3.8.1]. In particular, locally compact Hausdorff spaces are Čech-complete. The following characterization, due to Frolík [10], shows that Čech-complete spaces are strongly countably complete.

THEOREM 1.1. A Tychonoff space $X$ is Čech-complete if and only if there is a sequence $\left\{\mathscr{A}_{i}: i=1,2, \cdots\right\}$ of open coverings of $X$ such that, if a family $\mathscr{F}$ of closed subsets of $X$ has the finite intersection property, and if $\mathscr{F}$ contains $\mathscr{A}_{i}$-small members for each $i$, then $\cap \mathscr{F} \neq \varnothing$.

It follows from the theorem that each topological space which is homeomorphic to a complete metric space is Cech-complete. The product of a countable family of Čech-complete spaces is again Čechcomplete [8, Theorem 3.8.5]. For additional permanence properties, consult [8]. The proof of the Baire category theorem in [8] actually shows that a strongly countably complete regular space is Baire.

A map $f$ of the product $X \times Y$ of topological spaces $X$ and $Y$ into a topological space $Z$ is said to be separately continuous if, for each $\left(x_{0}, y_{0}\right)$ in $X \times Y$, the maps $x \mapsto f\left(x, y_{0}\right): X \rightarrow Z$ and $y \mapsto f\left(x_{0}\right.$, $y): Y \rightarrow Z$ are continuous. When $f$ is continuous at $\left(x_{0}, y_{0}\right)$ relative to the product topology, we shall say that $f$ is jointly continuous at $\left(x_{0}, y_{0}\right)$ for emphasis. The following theorem gives a relationship between separate continuity and joint continuity, and it is the fundamental theorem of the present paper.

THEOREM 1.2. Let $X$ be a strongly countably complete regular space, let $Y$ be a locally compact and $\sigma$-compact space, and let $Z$ be a pseudo-metrizable space. If a map $f: X \times Y \rightarrow Z$ is separately continuous, then there exists a dense $G_{\dot{o}}$-set $A$ in $X$, such that $f$ is jointly continuous at each point of $A \times Y$.

Proof. We may obviously assume that $X \neq \varnothing$ and $Y \neq \varnothing$. Now it is easy to deduce the theorem from the special case where $Y$ is compact. For, under the hypothesis on $Y$, there is a sequence $\left\{Y_{i}: i=1,2, \cdots\right\}$ of compact subsets of $Y$ such that $Y=U\{$ Int $\left.Y_{i}: i=1,2, \cdots\right\}$. Suppose that, for each $i$, we can find a dense $G_{\tilde{o}}$-set 
$A_{i}$ in $X$ such that $f \mid X \times Y_{i}$ is jointly continuous at each point of $A_{i} \times Y_{\imath}$. Then $f$ is jointly continuous at each point of $\left(\bigcap_{i} A_{i}\right) \times Y$, and $\bigcap_{i} A_{i}$ is a dense $G_{o}$ in $X$, because $X$ is Baire. Therefore, for the rest of the proof, we shall assume that $Y$ is compact. The proof is divided into four steps.

I. Let $d$ be a pseudo-metric for $Z$ compatible with the topology, and, for a positive number $\varepsilon$, let $O_{\varepsilon}$ be the union of all open subsets $O$ of $X \times Y$ such that $\operatorname{diam} f[O] \leqq \varepsilon$, where, for a subset $S$ of $Z$, $\operatorname{diam} S=\sup \{d(u, v): u, v \in S\} . \quad$ Clearly $O_{\varepsilon}$ is open in $X \times Y$. Let $A_{\varepsilon}=\left\{x:\{x\} \times Y \subset O_{\varepsilon}\right\}=X \sim p\left[X \times Y \sim O_{\varepsilon}\right]$, where $p: X \times Y \rightarrow X$ is the projection of the product space onto the first factor. Since $Y$ is compact, $p$ is a closed map. Therefore, $A_{\varepsilon}$ is an open subset of $X$. Let $A=\bigcap\left\{A_{1 / n}: n=1,2, \cdots\right\}$ and $D=\bigcap\left\{O_{1 / n}: n=1,2, \cdots\right\}$. Then $D$ is the set of points of joint continuity of $f$ and $A \times Y \subset D$. If it is shown that $A_{\varepsilon}$ is dense in $X$ for each positive number $\varepsilon$, then $A$ is a dense $G_{\delta}$ in $X$, because $X$ is Baire. Therefore, it remains to prove that $A_{\varepsilon}$ is dense in $X$.

Let $U$ be a nonempty open subset of $X$, and let $\varepsilon$ be a fixed positive number. We will show that $U \cap A_{\varepsilon} \neq \varnothing$ by contradiction. Assume, then, that $U \cap A_{\varepsilon}=\varnothing$, or equivalently that $U \subset p\left[X \times Y \sim O_{\varepsilon}\right]$. Let $F$ be a minimal closed subset of $X \times Y \sim O_{\varepsilon}$ such that $U \subset p[F]$. Such a minimal closed subset exists. For, suppose that $\left\{F_{\alpha}\right\}$ is a nest of closed subsets of $X \times Y \sim O_{\varepsilon}$ such that $U \subset p\left[F_{a}\right]$ for each $\alpha$. For each $x$ in $U$, the family $\left\{(\{x\} \times Y) \cap F_{\alpha}\right\}$ of compact sets has the finite intersection property, and therefore $(\{x\} \times Y) \cap \bigcap_{\alpha} F_{\alpha} \neq \varnothing$ for each $x$ in $U$, i.e., $U \subset p\left[\bigcap_{\alpha} F_{\alpha}\right]$. Zorn's lemma can now be applied to ensure the existence of a minimal $F$.

II. Let $\delta=\varepsilon / 6$. In this step, we shall establish the following property of the set $F$ constructed in step I.

( $\star$ Let $\left(x_{0}, y_{0}\right) \in F$, let $V$ be an open neighborhood of $x_{0}$ in $X$, and let $W$ be an open neighborhood of $y_{0}$ in $Y$. Then there exist $u$ in $V$ and $v, w$ in $W$ such that

$$
(u, w) \in F \quad \text { and } \quad d(f(u, v), f(u, w)) \geqq \delta .
$$

Proof of ( $\star$. By the minimality of $F$, we know that $U \subset p[F]$ and $U \not \subset p[F \sim(V \times W)]$. Let $\bar{x} \in U \sim p[F \sim(V \times W)]$. Then clearly $\bar{x} \in U \cap V$. Since $p[F \sim(V \times W)]$ is closed, there is an open neighborhood $N$ of $\bar{x}$ such that $\bar{x} \in N \subset U \cap V$ and $N \cap p[F \sim(V \times W)]=\varnothing$. Then, for each $x$ in $N, \varnothing \neq\{y:(x, y) \in F\} \subset W$. For each $x$ in $N$, choose an element $\sigma(x)$ in $W$ so that $(x, \sigma(x)) \in F$, and write $\bar{y}=\sigma(\bar{x})$. 
By making $N$ smaller, if necessary, we may assume that $d(f(x, \bar{y})$, $f(\bar{x}, \bar{y}))<\delta$ whenever $x \in N$. Suppose for a moment that $d(f(x, y)$, $f(x, \sigma(x)))<\delta$ for all $(x, y)$ in $N \times W$. This would imply that, for each $(x, y)$ in $N \times W$,

$$
\begin{aligned}
d(f(x, y), f(\bar{x}, \bar{y})) \leqq & d(f(x, y), f(x, \sigma(x)))+d(f(x, \bar{y}), f(x, \sigma(x))) \\
& +d(f(x, \bar{y}), f(\bar{x}, \bar{y}))<3 \delta=\varepsilon / 2
\end{aligned}
$$

It follows that diam $f[N \times W] \leqq \varepsilon$ and hence $N \times W \subset O_{\varepsilon}$, but this contradicts the fact that $(\bar{x}, \bar{y}) \in N \times W$ and $(\bar{x}, \bar{y})=(\bar{x}, \sigma(\bar{x})) \in F \subset$ $X \times Y \sim O_{\varepsilon}$. Therefore there must exist $(u, v)$ in $N \times W$ such that $d(f(u, v), f(u, \sigma(u))) \geqq \delta$. Let $w=\sigma(u)$; the $u, v, w$ satisfy all the requirements of property $(\star)$.

III. Since the space $X$ is strongly countably complete, there is a sequence $\left\{\mathscr{A}_{i}: i=1,2, \cdots\right\}$ of open coverings of $X$ that has the property: If $\left\{C_{i}\right\}$ is a sequence of closed subsets of $X$ such that $C_{i} \supset C_{i+1}$ and $C_{i}$ is $\mathscr{A}_{i}$-small for all $i$, then $\bigcap_{i} C_{i} \neq \varnothing$. By induction we construct sequence $\left\{x_{i}\right\}$ in $U$ and $\left\{y_{\imath}\right\},\left\{z_{2}\right\}$ in $Y$ and a sequence $\left\{G_{i}\right\}$ of open subsets of $X$ such that

(i) $\left(x_{i}, z_{i}\right) \in F$ for all $i$,

(ii) $d\left(f\left(x_{j}, y_{i}\right), f\left(x_{i}, y_{i}\right)\right)<\delta / 3$ for $i<j$,

(iii) $d\left(f\left(x_{i}, y_{j}\right), f\left(x_{i}, z_{i}\right)\right)<\delta / 3$ for $i<j$,

(iv) $d\left(f\left(x_{i}, z_{j}\right), f\left(x_{i}, z_{2}\right)\right)<\delta / 3$ for $i<j$,

(v) $d\left(f\left(x_{i}, y_{i}\right), f\left(x_{i}, z_{i}\right)\right) \geqq \delta$ for all $i$,

(vi) $x_{j} \in G_{i}$ for $i<j$, and

(vii) $\bar{G}_{i}$ is $\mathscr{L}_{i}$-small for all $i$.

Let $\left(x_{0}, y_{0}\right)$ be any point in $F$ such that $x_{0} \in U$, and apply property ( $*$ with $V=U$ and $W=Y$. Then we can find $x_{1}$ in $U$ and $y_{1}, z_{1}$ in $Y$ satisfying (i) and (v) for $i=1$. Next assume that $x_{1}, \cdots, x_{n}$ in $U, y_{1}, \cdots, y_{n} ; z_{1}, \cdots, z_{n}$ in $Y$ and open subsets $G_{1}, \cdots, G_{n-1}$ of $X$ have been chosen so that (i)-(vi) are valid for $i \leqq n, j \leqq n$ and (vii) is valid for $i<n$. Let

$$
V_{n+1}=\left\{x: d\left(f\left(x, y_{i}\right), f\left(x_{\imath}, y_{\imath}\right)\right)<\delta / 3 \text { for } i=1, \cdots n,\right\} \cap U
$$

and

$$
W_{n+1}=\left\{w: d\left(f\left(x_{i}, w\right), f\left(x_{i}, z_{i}\right)\right)<\delta / 3 \text { for } i=1, \cdots, n\right\} \text {. }
$$

The sets $V_{n+1}$ and $W_{n+1}$ are open because $f$ is separately continuous. By inductive hypotheses (i), (ii), (iv), and (vi), we have $\left(x_{n}, z_{n}\right) \in F$, $x_{n} \in V_{n+1}, z_{n} \in W_{n+1}$ and $x_{n} \in G_{1} \cap \cdots \cap G_{n-1}$. Since $\mathscr{A}_{n}$ is an open covering of $X$ and since $X$ is regular, there is an open neighborhood $G_{n}$ of $x_{n}$ such that $\bar{G}_{n}$ is $\mathscr{A}_{n}$-small. Let $V_{n+1}^{*}=V_{n+1} \cap G_{1} \cap \cdots \cap G_{n}$. By property ( $\star$ ) applied to the open neighborhoods $V_{n+1}^{*}$ and $W_{n+1}$ of $x_{n}$ 
and $z_{n}$ respectively, we can choose $x_{n+1}$ in $V_{n+1}^{*}$ and $y_{n+1}, z_{n+1}$ in $W_{n+1}$ so that $\left(x_{n+1}, z_{n+1}\right) \in F$ and $d\left(f\left(x_{n+1}, y_{n+1}\right), f\left(x_{n+1}, z_{n+1}\right)\right) \geqq \delta$. Then (i)(vi) hold for $i \leqq n+1, j \leqq n+1$, and (vii) holds for $i \leqq n$, and therefore the inductive construction is complete.

IV. Now we prove that the construction of step III leads to a contradiction. Observe first that (ii), (iii), and (v) imply

(viii) $d\left(f\left(x_{j}, y_{i}\right) f\left(x_{i}, y_{j}\right)\right)>\delta / 3$ for $i<j$.

Next, let $C_{i}=\left\{x_{j}: j>i\right\}^{-}$for $i=1,2, \cdots$. Then clearly $C_{i} \supset C_{i+1}$ for $i=1,2, \cdots$, and by (vi) and (vii) the closed set $C_{i}$ is $\mathscr{A}_{i}$-small for each $i$. Therefore, by the property of $\mathscr{A}_{i}$ stated in step III, $\bigcap_{i} C_{i} \neq \varnothing$, i.e., the sequence $\left\{x_{j}: j=1,2, \cdots\right\}$ has a cluster point. Since the sequence $\left\{y_{j}: j=1,2, \cdots\right\}$ lies in a compact set $Y$, the sequence $\left\{\left(x_{j}, y_{j}\right): j=\right.$ $1,2, \cdots\}$ has a cluster point in $X \times Y$, say $(a, b)$.

For a fixed $i$, the map $(x, y) \mapsto d\left(f\left(x, y_{i}\right), f\left(x_{i}, y\right)\right)$ is continuous on $X \times Y$. Therefore (viii) implies that

(ix) $d\left(f\left(a, y_{i}\right), f\left(x_{i}, b\right)\right) \geqq \delta / 3$ for all $i$.

Similarly the map $(x, y) \mapsto d(f(a, y), f(x, b))$ is continuous. Hence (ix) implies that $d(f(a, b), f(a, b)) \geqq \delta / 3$. But this last inequality is absurd; hence the assumption in step I that $U \cap A_{\varepsilon}=\varnothing$ is untenable, and the proof is complete.

REMARKs 1.3. (a) In some specific cases, the proof of Theorem 1.2 can be made much simpler. For instance, assume that $X$ is locally countably compact and regular. Then in step $I, \bar{U}$ can be assumed to be countably compact without loss of generality. Then in step III, construct sequences $\left\{x_{i}\right\},\left\{y_{i}\right\},\left\{z_{i}\right\}$ satisfying (i)-(v), and disregard $\left\{\mathscr{A}_{i}\right\}$ and conditions (vi), (vii). Here $V_{n+1}$ and $W_{n+1}$ are all we need for the construction. In step IV, the existence of a cluster point of the sequence $\left\{\left(x_{i}, y_{i}\right)\right\}$ is immediate, because the sequence lies in a countably compact set $\bar{U} \times Y$.

(b) In [9], Fort proved the following theorem that is not strictly comparable to Theorem 1.2 but similar in form: Let $X$ be a topological space, let $Y$ be a locally compact separable metric space, and lel $Z$ be a separable metric space. If $f: X \times Y \rightarrow Z$ is separately centinuous then there is a residual subset $A$ of $X$ such that $f$ is jointly continuous at each point of $A \times Y$. In view of Fort's theorem, it is natural to ask if Theorem 1.2 remains valid when $X$ is only assumed to be Baire. We are unable to answer this question.

2. Function spaces. It is often convenient to reformulate Theorem 1.2 in terms of function spaces. The following lemma is useful in this connection. An easy proof is omitted. 
Lemma 2.1. Let $X$ be a topological space, let $Y$ be a compact space, and let $(Z, d)$ be a pseudo-metric space. Suppose that a function $f: X \times Y \rightarrow Z$ is jointly continuous at each point of $\left\{x_{0}\right\} \times Y$ for some $x_{0}$ in $X$. Then for each positive number $\varepsilon$, there is a neighborhood $U$ of $x_{0}$ such that $d\left(f(x, y), f\left(x_{0}, y\right)\right) \leqq \varepsilon$ for all $x$ in $U$ and all $y$ in $Y$.

For topological spaces $Y$ and $Z, C(Y, Z)$ denotes the space of all continuous maps on $Y$ into $Z$. In case $Z$ is the space $\boldsymbol{R}$ of real numbers, $C(Y, \boldsymbol{R})$ will simply be denoted by $C(Y)$. The pointwise topology (i.e., the topology of pointwise convergence) for $C(Y, Z)$ is denoted by $\mathscr{P}$. If $Z$ is a pseudo-metric (or uniform) space and if $\mathscr{A}$ is a family of subsets of $Y$, then the topology of uniform convergence on members of $\mathscr{A}$ is denoted by $\mathscr{T}_{\mathscr{A}}$.

THEOREM 2.2. Let $X$ be a strongly countably complete regular space, let $\mathscr{A}$ be a countable family of compact subsets of a topological space $Y$ such that $\mathrm{U} \mathscr{A}=Y$, and let $(Z, d)$ ba a pseudo-metric space. If $f$ is a continuous map of $X$ into $(C(Y, Z), \mathscr{P})$, then there is a dense $G_{\hat{o}}$-set $A$ in $X$ such that, at each point of $A, f$ is continuous from $X$ to $\left(C(Y, Z), \mathscr{T}_{\mathscr{s}}\right)$.

Proof. Let $\mathscr{A}=\left\{Y_{i}: i=1,2, \cdots\right\}$, and for each $i$ let $F_{i}: X \times$ $Y_{i} \rightarrow Z$ be the map given by $F_{i}(x, y)=f(x)(y)$. Then obviously $F_{i}$ is separately continuous. By Theorem $1.2, F_{i}$ is jointly continuous at each point of $A_{i} \times Y_{\imath}$ for some dense $G_{\tilde{o}}$-set $A_{i}$ in $X$. Let $A=$ $\bigcap_{i} A_{i}$; then $A$ is a dense $G_{\delta}$ in $X$. It follows from Lemma 2.1 that, for each $x_{0}$ in $A$, each positive $\varepsilon$, and for each positive integer $i$, there is a neighborhood $U$ of $x_{0}$ such that $\sup \left\{d\left(f(x)(y), f\left(x_{0}\right)(y)\right): y \in Y_{i}\right\} \leqq \varepsilon$ whenever $x \in U$. Therefore, $f$ is continuous relative to $\mathscr{T}_{\mathscr{A}}$ at $x_{0}$

Let $X$ be a topological space and let $(Z, d)$ be a pseudo-metric space. Recall that a subset $H$ of $C(X, Z)$ is equicontinuous at a point $x_{0}$ of $X$ if, for each positive number $\varepsilon$, there is a neighborhood $U$ of $x_{0}$ such that $d\left(h(x), h\left(x_{0}\right)\right) \leqq \varepsilon$ for all $h$ in $H$ and all $x$ in $U$. If $H$ is equicontinuous at each point of $X$, then $H$ is said to be equicontinuous. The following theorem is of interest when it is compared with Ascoli's theorem.

THEOREM 2.3. Let $X$ be a strongly countably complete regular space, and let $Z$ be pseudo-metric space. If a subset $H$ of $C(X, Z)$ is compact relative to the pointwise topology, then $H$ is equicontinuous at each point of a dense $G_{i}$-set in $X$.

Proof. Let $H$ be provided with the pointwise topology; then by 
assumption $H$ is a compact space. The map $f: X \times H \rightarrow Z$ given by $f(x, h)=h(x)$ is obviously separately continuous. By Theorem 1.2, there is a dense $G_{i}$-set $A$ in $X$ such that $f$ is jointly continuous at each point of $A \times H$. Then by Lemma 2.1 and by the definition of equicontinuity, the family $H$ is equicontinuous at each point of $A$.

The following corollary is now obvious.

CoRollary 2.4. Let $X$ be a compact Hausdorff space, and let $C(X)$ be the Banach space of all continuous real-valued functions on $X$ with the supremum norm. If a subset $H$ of $C(X)$ is weakly compact, then $H$ is equicontinuous at each point of a dense $G_{\dot{o}}$-set in $X$.

REMARK 2.5. Let $C(X)$ be as in Corollary 2.4. Then by Ascoli's theorem, a bounded subset $H$ of $C(X)$ is relatively compact (i.e., the closure of $H$ is compact) with respect to the norm topology if and anly if $H$ is equicontinuous. In view of Corollary 2.4, one is tempted to conjecture that a bounded subset $H$ of $C(X)$ is weakly relatively compact if and only if $H$ is equicontinuous at each point of a dense $G_{\delta}$ in $X$. Unfortunately this is false. For, suppose $\left\{f_{i}: i=1,2, \cdots\right\}$ is a uniformly bounded sequence of continuous real-valued functions on $[0,1]$ such that $f(x)=\lim _{i} f_{i}(x)$ exists for each $x$ in $[0,1]$ and that the limit function $f$ is not continuous. Then by the Osgood theorem [14, Theorem 9.5], the family $\left\{f_{i}\right\}$ is equicontinuous at each point of a dense $G_{\delta}$ in $[0,1]$. However, $\left\{f_{i}\right\}$ connot be weakly relatively compact in $C([0,1])$, since the limit function $f$ is not continuous.

3. Group actions. When the theorems of previous sections are combined with group actions, some strong theorems should emerge because the group actions spread the points of continuity around. We shall illustrate this principle by proving two theorems. The first theorem is a generalization of a classic theorem by Ellis [7, Theorem 1], and the second one is a slightly weaker version of a theorem enunciated by Corson and Glicksberg [6, Theorem 1] (see however, Remark 3.4).

A group $G$ is said to act on a topological space $X$ if a map: $G \times X \rightarrow X$ (denoted by $(g, x) \mapsto g \cdot x)$ is given so that the following conditions are satisfied:

(a) the map $x \mapsto g \cdot x$ is continuous for each $g$ in $G$, and

(b) $g \cdot(h \cdot x)=(g h) \cdot x$ for all $g, h$ in $G$ and $x$ in $X$.

(c) If $e$ denotes the identity element of $G$, then $e \cdot x=x$ for all $x \in X$.

Of course, (a)-(c) imply that, for each $g$, the map $x \mapsto g \cdot x$ is a homeomorphism of $X$ onto itself. 
THEOREM 3.1. Let $X$ be a locally compact regular space and let $G$ be a group acting on $X$. Suppose that $G$ is given a topology such that:

(i) $G$ is strongly countably complete and regular,

(ii) the $\operatorname{map}(g, x) \mapsto g \cdot x: G \times X \rightarrow X$ is separately continuous, and

(iii) the $\operatorname{map} h \mapsto h g: G \rightarrow G$ is continuous for each $g$ in $G$. Then the map of (ii) is continuous (relative to the product topology).

Proof. Let $X^{+}$be the one-point compactification [13; p. 150] of $X$ with $\infty$ denoting the point at infinity. Then $X^{+}$is easily seen to be regular and, hence, completely regular [13, p. 146]. We can extend the action of $G$ on $X$ to $X^{+}$by defining $g \cdot \infty=\infty$ for all $g$ in $G$. Condition (ii) still holds when $X$ is replaced by $X^{+}$. Therefore, without loss of generality we may assume that $X$ is a compact regular space.

Since $X$ is completely regular, in order to prove that the map $(g, x) \mapsto g \cdot x: G \times X \rightarrow X$ is continuous, it is sufficient to prove that the map $(g, x) \mapsto \phi(g \cdot x): G \times X \rightarrow \boldsymbol{R}$ is continuous for each continuous real-valued function $\phi$ on $X$. Let $F: G \rightarrow C(X)$ be the map given by $F(g)(x)=\dot{\phi}(g \cdot x)$, and, for each $\alpha$ in $C(X)$ and $g$ in $G$, define a member $\alpha \cdot g$ of $C(X)$ by $(\alpha \cdot g)(x)=\alpha(g \cdot x)$. We note the following formal properties: The map $\alpha \mapsto \alpha \cdot g: C(X) \rightarrow C(X)$ is linear; $\|\alpha \cdot g\|=\|\alpha\|$ where $\|\cdot\|$ is the supremum norm; $(\alpha \cdot g) \cdot h=\alpha \cdot(g h)$ and $F(g h)=$ $F(g) \cdot h$ for all $\alpha$ in $C(X)$ and $g, h$ in $G$.

Now $F$ is clearly continuous with respect to the poinwise topology for $C(X)$. Hence, by Theorem 2.2, $F$ is continuous at some point $a$ of $G$ relative to the norm topology for $C(X)$. Let $b$ be an arbitrary point of $G$ and let $\left\{g_{\gamma}\right\}$ be a net in $G$ converging to $b$. Then by (iii), $g_{\gamma} b^{-1} a \rightarrow a$. Therefore, $\left\|F\left(g_{\gamma} b^{-1} a\right)-F(a)\right\| \rightarrow 0$. But

$$
\begin{aligned}
\left\|F\left(g_{\gamma}\right)-F(b)\right\| & =\left\|F\left(g_{\gamma} b^{-1} a\right) \cdot\left(a^{-1} b\right)-F(a) \cdot\left(a^{-1} b\right)\right\| \\
& =\left\|\left(F\left(g_{\gamma} b^{-1} a\right)-F(a)\right) \cdot\left(a^{-1} b\right)\right\| \\
& =\left\|F\left(g_{\gamma} b^{-1} a\right)-F(a)\right\| .
\end{aligned}
$$

It follows that $\left\|F\left(g_{\gamma}\right)-F(b)\right\| \rightarrow 0$ i.e., the map $F$ is continuous relative to the norm topology for $C(X)$. This, of course, implies that $(g, x) \mapsto F(g)(x)=\phi(g \cdot x): G \times X \rightarrow \boldsymbol{R}$ is continuous.

Let $G$ be a topological group. A $G$-space consists of a topological space $M$ and an action of the group $G$ on $M$ such that the map $(g, m) \mapsto g \cdot m: G \times M \rightarrow M$ is continuous. The topological group $G$ itself will be considered a $G$-space relative to the multiplication map: $G \times G \rightarrow G$. Given two $G$-spaces $M$ and $M_{1}$, a $G$-map $\pi: M \rightarrow M_{1}$ is a continuous map $\pi$ such that $\pi(g \cdot x)=g \cdot \pi(x)$ for all $g$ in $G$ and $x$ 
in $M$. In particular, a continuous map $\pi: G \rightarrow M$ is a $G$-map if $\pi(g h)=g \cdot \pi(h)$ for all $g, h$ in $G$.

Lemma 3.2. Let $G$ be a topological group. Then the topology of $G$ is determined by all G-maps of $G$ into pseudo-metrizable G-spaces, i.e., $a$ net $\left\{g_{\gamma}\right\}$ in $G$ converges to $g$ if and only if $\pi\left(g_{\gamma}\right) \rightarrow \pi(g)$ for all $G$-maps $\pi$ of $G$ into pseudo-metrizable $G$-spaces.

Proof. Let $U$ be an arbitrary neighborhood of the identity $e$ in $G$. Then one can construct a sequence $\left\{U_{n}: n=1,2, \cdots\right\}$ of symmetric open neighborhoods of $e$ such that $U_{1} \subset U$ and $U_{n+1} U_{n+1} \subset U_{n}$ for all $n$. For each $n$, let ${ }^{*} U_{n}=\left\{(x, y): y^{-1} x \in U_{n}\right\} \subset G \times G$. Then $\left\{{ }^{*} U_{n}: n=\right.$ $1,2, \cdots\}$ is a base for a uniformity $\mathscr{C}$ that is pseudo-metrizable. Let $d$ be a pseudo-metric for $G$ compatible with $\mathscr{U}$, and let $\mathscr{T}$ be the original topology of $G$. We assert that the map $(x, y) \mapsto x y:(G, \mathscr{T}) \times$ $(G, d) \rightarrow(G, d)$ is continuous. For, suppose that $\left\{g_{r}\right\}$ is a net in $G$ converging to $a$ relative to $\mathscr{T}$ and that $\left\{h_{\gamma}\right\}$ is a net in $G$ converging to $b$ relative to $d$. Then $\left\{b^{-1} h_{\gamma}\right\}$ is eventually in each $U_{n+1}$, and, since $\left\{a^{-1} g_{\gamma}\right\} \rightarrow e$ relative to $\mathscr{T},\left\{b^{-1} a^{-1} g_{\gamma} b\right\}$ is eventually in each $U_{n+1}$. Therefore, $\left\{(a b)^{-1} g_{\gamma} h_{r}\right\}=\left\{\left(b^{-1} a^{-1} g_{\gamma} b\right)\left(b^{-1} h_{\gamma}\right)\right\}$ is eventually in each $U_{n}$, i.e., $\left\{g_{\gamma} h_{\gamma}\right\} \rightarrow a b$ relative to $d$. This shows that $(G, d)$ is a pseudometrizable $G$-space. Obviously the identity map $(G, \mathscr{T}) \rightarrow(G, d)$ is a $G$-map. Therefore, there are enough such maps to determine the topology $\mathscr{T}$.

Given two topological groups $H$ and $G$, we denote by $\operatorname{Hom}(H, G)$ the space of all continuous homomorphisms of $H$ into $G$.

THEOREM 3.3. Let $H$ be a strongly countably complete topological group, and let $G$ be an arbitrary topological group. If a subset $F$ of $\operatorname{Hom}(H, G)$ is compact relative to the pointwise topology, then $F$ is compact relative to the compact open topology.

Proof. Provide the set $F$ with the pointwise topology so that $F$ is a compact space, and let $\phi: H \times F \rightarrow G$ be the map defined by $\phi(h, f)=f(h)$. Clearly $\phi$ is separately continuous, and we shall show that $\phi$ is continuous. In view of the lemma, it is sufficient to prove that $\pi \circ \phi: H \times F \rightarrow M$ is continuous, where $\pi: G \rightarrow M$ is an arbitrary $G$-map of $G$ into a pseudo-metrizable $G$-space $M$. Let $\psi=\pi \circ \phi$; then $\psi(h g, f)=f(h) \cdot \psi(g, f)$ for all $f$ in $F$ and $h, g$ in $H$. Since $\psi$ is separately continuous, it follows from Theorem 1.2 that there is a point $a$ of $G$ such that $\psi$ is jointly continuous at each point of $\{a\} \times F$. Now suppose that $\left\{\left(h_{r}, f_{r}\right)\right\}$ is a net in $H \times F$ converging to $(b, u)$. Then by the above remark, $\psi\left(h_{\gamma}, f_{\gamma}\right)=f_{\gamma}\left(b a^{-1}\right) \psi\left(a b^{-1} h_{\gamma}, f_{\gamma}\right)$. Since $a b^{-1} h_{\gamma} \rightarrow a, \psi\left(a b^{-1} h_{\gamma}, f_{\gamma}\right) \rightarrow \psi(a, u)$ by the choice of $a$. Also, since 
$f_{\gamma} \rightarrow u$ relative to the pointwise topology, $f_{\gamma}\left(b a^{-1}\right) \rightarrow u\left(b a^{-1}\right)$. Therefore, $\psi\left(h_{\gamma}, f_{r}\right) \rightarrow u\left(b a^{-1}\right) \psi(a, u)=\psi(b, u)$. This proves that $\phi$ is continuous. This shows that, on $F$, the pointwise topology is stronger than the compact open topology (see, for instance, Theorem 5 of [13, p. 223]). It follows that $F$ is compact relative to the compact open topology.

REMARK 3.4. In [6], Corson and Glicksberg state a theorem stronger than Theorem 3.3. They require $H$ to satisfy only the following condition: Each closed subgroup of $H$ is Baire. Clearly if $H$ is strongly countably complete, it satisfies the condition of Corson and Glicksberg, and the converse is probably false. It should be pointed out, however, that the proof of Corson and Glicksberg is incomplete, because they assumd that the topology of an arbitrary topological group $G$ is determined by a family of homomorphisms of $G$ into metrizable topological groups. It is very likely that their proof can be repaired by using something like Lemma 3.2, but, at any rate, their proof remains valid for a large class of topological groups. Caution: In the discussion above, the roles of $H$ and $G$ are exactly the reverse of those in [6]. We avoided the term " $H$-space", since it has quite a different connotation among some topologists.

4. Weak and weak* topologies. Let $E$ be a locally convex metrizable linear topological space, then there is a countable family $\mathscr{A}$ of weak*-compact subsets of the dual $E^{*}$ such that $\cup \mathscr{A}=E^{*}$ and that the topology of $E$ is identical with $\mathscr{T}_{\mathscr{A}}$ where $E$ is viewed as a space of weak*-continuous functions on $E^{*}$. Hence the following theorem is an immediate consequence of Theorem 2.2.

THEOREM 4.1. Let $X$ be a strongly countably complete regular space, and let $(E, \mathscr{T})$ be a locally convex metrizable linear topological space. If $f: X \rightarrow(E$, weak $)$ is a continuous map, then there is a dense $G_{\tilde{o}}$-set $A$ in $X$ such that, at each point of $A, f$ is continuous from $X$ to $(E, \mathscr{T})$.

If $K$ is a subset of a topological space $(E, \mathscr{T})$, we denote by $(K, \mathscr{T})$ the space $K$ with the topology induced by $\mathscr{T}$.

COROLlary 4.2. Let $K$ be a weak-compact subset of a locally convex metrizable linear topological space $(E, \mathscr{T})$. Then there is a dense $G_{\dot{o}}$-set $A$ in $(K$, weak) such that the identity map (K, weak) $\rightarrow$ $(K, \mathscr{T})$ is continuous at each point of $A$. In particular each point of $A$ is a $G_{\delta}$-point in (K, weak).

A weak-compact subset of a locally convex metrizable linear 
topological space is 'Eberlein compact' in the sense of [1]. Amir and Lindenstrauss proved that in an Eberlein compact set the set of all $G_{i}$-points is dense [1].

In Corollary 4.1, if $K$ is further assumed to be convex, then $A \cap \operatorname{ext}(K)$ is weak-dense in ext $(K)$, where ext $(K)$ is the set of all extreme points of $K$. We prove this fact by the methods of [18]. The necessary technical result is contained in [18, Theorem 2.3], which we quote here with a slight modification to suit our purpose.

THEOREM 4.3. Let $\mathscr{T}_{1}$ and $\mathscr{T}_{2}$ be two locally convex vector topologies for a linear space $E$ such that

(i) there is a local base for $\mathscr{T}_{1}$ consisting of $\mathscr{T}_{2}$-closed sets,

(ii) $\left(E, \mathscr{T}_{1}\right)$ is pseud-metrizable and $\left(E, \mathscr{T}_{2}\right)$ is Hausdorff, and

(iii) for each $\mathscr{T}_{2}$-compact subset $D$ of $E$, the identity map: $\left(D, \mathscr{T}_{2}\right) \rightarrow\left(D, \mathscr{T}_{1}\right)$ is continuous at each point of a dense subset of $\left(D, \mathscr{T}_{2}\right)$.

Then, if $K$ is a $\mathscr{T}_{2}$-compact convex subset of $E$ and if $Z$ is the set of all points of continuity of the identity map: $\left(K, \mathscr{T}_{2}\right) \rightarrow\left(K, \mathscr{T}_{1}\right)$, the intersection $Z \cap \operatorname{ext}(K)$ is $\mathscr{T}_{2}$-dense in ext $(K)$, and $K$ is the $\mathscr{T}_{2}$-closed convex hull of $Z \cap \operatorname{ext}(K)$.

Proof. We only indicate the necessary modification of the proof in [18]. ${ }^{1} \quad$ First assume that the topology $\mathscr{T}_{1}$ is defined by a single lower $\mathscr{T}_{2}$-semicontinuous pseudo-norm $p$. Then follow the proof of [18, Theorem 2.2] verbatim except that " $\mathscr{T}_{p}$ " and " $\mathscr{T}$ " should be replaced by " $\mathscr{T}_{1}$ " and " $\mathscr{T}_{2}$ " respectively. The separability of $\left(E, \mathscr{T}_{1}\right)$ and [18, Proposition 1.2] are not necessary here because of assumption (iii). The proof of [18, Theorem 2.3] can now be used verbatim. This concludes the proof.

Now in Theorem 4.3 , let $\left(E, \mathscr{T}_{1}\right)$ be a locally convex metrizable linear topological space and let $\mathscr{T}_{2}$ be the weak topology $\left(=w\left(E, E^{*}\right)\right)$. Then assumptions (i) and (ii) are clearly satisfied, and Corollary 4.2 states that assumption (iii) is also satisfied. Therefore we obtain:

THeOREM 4.4. Let $(E, \mathscr{T})$ be a locally convex metrizable linear topological space, let $K$ be a weak-compact convex subset of $E$, and let $A$ be the set of all points of continuity of the identity map ( $K$, weak) $\rightarrow(K, \mathscr{T})$. Then $A \cap \operatorname{ext}(K)$ is weak-dense in ext $(K)$, and $K$ is the closed convex hull of $A \cap \operatorname{ext}(K)$.

Theorem 4.4 was proved in [18] with the extra condition that

${ }^{1}$ We take this opportunity to make the following correction in [18]. Replace "of the 2nd category in itself" by "Baire" at the following three places: p. 147 line 3; p. 148 line 2 ; p. 169 line 6. 
$(E, \mathscr{T})$ be separable. In case $(E, \mathscr{T})$ is a Banach space, Theorem 4.4 is a consequence of Troyanski's theorem [21, Corollary 7] that depends on many difficult theorems in Banach space theory due to Troyanski, Amir-Lindenstrauss and Lindenstrauss. In contrast with Troyanski's proof, where norms of a particular kind play an essential role, our approach is completely topological and relatively easy.

Remark 4.5. Let $(E, \mathscr{T}), K$ and $A$ be as in Theorem 4.4, let $u \in A \cap \operatorname{ext}(K)$, and let $U$ be an arbitrary $\mathscr{T}$-neighborhood of $u$. Then, by the definition of $A$, there is a weak-open neighborhood $W$ of $u$ such that $W \cap K \subset U$. Let $K_{0}$ be the closed convex hull of the weak-compact set $K \sim W$. If $u \in K_{0}$, then $u$ is extreme in $K_{0}$, and hence $u \in K \sim W$, contradicting the fact that $u \in W$. Therefore, $u \notin K_{0}$, and a fortiori $u$ is not contained in the closed convex hull of $K \sim U$. In case $(E, \mathscr{T})$ is a Banach space, a point of $K$ having the property just described is called a denting point of $K$. More specifically, a point $u$ of a subset $S$ of a Banach space is called a denting point of $S$ [20], if, for each positive $\varepsilon, u$ is not in the closed convex hull of $S \sim\{x:\|x-u\|<\varepsilon\}$. Hence Theorem 4.4 implies that each weak-compact convex subset of a Banach space is the convex closed hull of its denting points. When the Banach space is separable, this fact was observed by Rieffel [20] using a theorem of Lindenstrauss [15], and the general case is due to Troyanski [21].

Now we consider the weak* topology in the dual $E^{*}$ of a Banach space $E$. The situation here is much more complicated. Given a weak*-compact subset $K$ of $E^{*}$, there may not exist any point where the identity map $\left(K\right.$, weak $\left.{ }^{*}\right) \rightarrow(K$, norm $)$ is continuous. However, if $E^{*}$ is separable, then by an easy category argument one can prove that the identity map $\left(K\right.$, weak $\left.^{*}\right) \rightarrow(K$, norm $)$ is continuous at points of a weak*-dense subset of $K$ (see [18, Corollary 1.3]). We shall extend this result to the duals $E^{*}$ that are "weak-compactly generated" (for definition, see below).

Lemma 4.6. Let $E$ be a normed linear space, let $K$ be a nonempty weak* $\left(=w\left(E^{*}, E\right)\right)$-compact subset of $E^{*}$ such that $K \subset C+B_{\varepsilon}$ for some weak $\left(=w\left(E^{*}, E^{* *}\right)\right)$-compact subset $C$ of $E^{*}$ and for some positive number $\varepsilon$, where $B_{\varepsilon}=\left\{f: f \in E^{*},\|f\| \leqq \varepsilon\right\}$, and let $\delta>2 \varepsilon$. Then there is a nonempty open subset $W$ of $\left(K\right.$, weak $\left.{ }^{*}\right)$ such that $\operatorname{diam} W \leqq \delta$.

Proof. The space $K \times B_{\varepsilon}$ is compact with respect to the product of the weak* topologies, which will be again referred to as the weak* topology. Let $d: K \times B_{\varepsilon} \rightarrow E^{*}$ be the map defined by $d(f, g)=f-g$. 
Let $F=d^{-1}[C]$, and let $p: K \times B_{\varepsilon} \rightarrow K$ be the projection. Since $C$ is weak-compact, it is also weak*-compact, and consequently $C$ is weak*closed in $E^{*}$. Hence $F$ is weak ${ }^{*}$-closed in $K \times B_{\varepsilon}$, and $p[F]=K$ because $K \subset C+B_{\varepsilon}$. By using the weak*-compactness of $F$ and Zorn's lemma, we note that there is a minimal weak*-compact subset $F_{0}$ of $F$ such that $p\left[F_{0}\right]=K$ (cf. step I of the proof of Theorem 1.2). The map $d:\left(F_{0}\right.$, weak $\left.{ }^{*}\right) \rightarrow\left(d\left[F_{0}\right]\right.$, weak $)$ is continuous and $d\left[F_{0}\right]$ is weakcompact, because the weak and weak* topologies coincide on $C$. By Corollary 4.2, the identity map $i:\left(d\left[F_{0}\right]\right.$, weak $) \rightarrow\left(d\left[F_{0}\right]\right.$, norm $)$ is continuous at some point of $d\left[F_{0}\right]$. Therefore, there is a point $\left(f_{0}, g_{0}\right) \in F_{0}$ where $i \circ d:\left(F_{0}\right.$, weak $\left.{ }^{*}\right) \rightarrow\left(d\left[F_{0}\right]\right.$, norm $)$ is continuous. It follows that there is an open neighborhood $V$ of $\left(f_{0}, g_{0}\right)$ in $\left(K \times B_{\varepsilon}\right.$, weak $\left.{ }^{*}\right)$ such that diam $d\left[F_{0} \cap V\right] \leqq \delta-2 \varepsilon$. By the minimality of $F_{0}, K \neq p\left[F_{0} \sim V\right]$. Therefore, $W=K \sim p\left[F_{0} \sim V\right]$ is a nonempty open subset of ( $K$, weak*). Let $f_{1}, f_{2} \in W$. Then there are $g_{1}, g_{2}$ in $B_{\varepsilon}$ such that $\left(f_{1}, g_{1}\right)$, $\left(f_{2}, g_{2}\right) \in F_{0}$. By the definition of $W$, it follows that $\left(f_{1}, g_{1}\right),\left(f_{2}, g_{2}\right) \in$ $F_{0} \cap V$. Therefore $\delta-2 \varepsilon \geqq\left\|d\left(f_{1}, g_{1}\right)-d\left(f_{2}, g_{2}\right)\right\|=\left\|f_{1}-g_{1}-f_{2}+g_{2}\right\|$, whence $\left\|f_{1}-f_{2}\right\| \leqq(\delta-2 \varepsilon)+\left\|g_{1}-g_{2}\right\| \leqq \delta$. This shows that diam $W \leqq \delta$, and the proof is complete.

A closed linear subspace $H$ of a Banach space $E$ is said to be weak-compactly generated if there is a weak-compact subset of $H$ whose linear extension is dense in $H$. Equivalently, $H$ is weakcompactly generated if and only if there is a weak-compact convex circled subset $C$ of $H$ such that $H$ is the closure of $\bigcup\{n C: n=1,2$, $\cdots\}$.

THEOREM 4.7. Let $E$ be a normed linear space, and let $K$ be a weak*-compact subset of $E^{*}$ such that $K \subset H$ for some weakcompactly generated norm-closed linear subspace $H$ of $E^{*}$. Then the identity map: $\left(K\right.$, weak $\left.{ }^{*}\right) \rightarrow(K$, norm $)$ is continuous at each point of a dense subset of (K, weak $\left.{ }^{*}\right)$.

Proof. Let $\varepsilon$ be a positive number and let $O_{\varepsilon}$ be the union of all open subsets $O$ of $\left(K\right.$, weak $\left.{ }^{*}\right)$ such that $\operatorname{diam} O \leqq \varepsilon$. Since $(K$, weak*) is a Baire space, it is sufficient to show that $O_{\varepsilon}$ is dense in $\left(K\right.$, weak ${ }^{*}$. Clearly we may assume that $X \neq \varnothing$. Let $U$ be an arbitrary nonempty open subset of $\left(K\right.$, weak $\left.{ }^{*}\right)$. Then we must prove that $O_{\varepsilon} \cap U \neq \varnothing$. Let $C$ be a weak-compact subset of $E^{*}$ such that $\mathrm{U}\{n C: n=1,2, \cdots\}$ is norm-dense in $H$, and let $\delta=\varepsilon / 3$. Then $U \subset \bigcup\left\{n C+B_{\delta}: n=1,2, \cdots\right\}$. Since each $n C+B_{\delta}$ is weak*-closed in $E^{*}$ and since $\left(U\right.$, weak $\left.{ }^{*}\right)$ is a Baire spaces, there is a nonempty open subset $V$ in $\left(K\right.$, weak $\left.^{*}\right)$ and a positive integer $n$ such that $V^{-} \subset U \cap\left(n C+B_{\delta}\right)$, where $V^{-}$is the weak ${ }^{*}$-closure of $V$ in $K$. Since $V^{-}$is nonempty and weak*-compact, and since $n C$ is weak-compact, 
it follows from Lemma 4.6 that there is a nonempty open subset $W$ of $\left(V^{-}\right.$, weak $\left.{ }^{*}\right)$ such that diam $W \leqq 3 \delta=\varepsilon$. Since $V$ is weak ${ }^{*}$-dense in $V^{-}, V \cap W$ is nonempty. Also $V \cap W$ is open in $\left(V\right.$, weak $\left.{ }^{*}\right)$ and, hence, in $\left(K\right.$, weak $\left.{ }^{*}\right)$. Therefore, $\varnothing \neq V \cap W \subset O_{\varepsilon} \cap U$, and the proof is complete.

THEOREM 4.8. Let $E$ be a normed linear space, and let $K$ be a weak*-compact convex subset of $E^{*}$ such that $K \subset H$ for some weakcompactly generated, norm-closed, linear subspace $H$ of $E^{*}$. If $A$ is the set of all points of continuity of the identity map: $\left(K\right.$, weak $\left.^{*}\right) \rightarrow$ ( $K$, norm), then $A \cap \operatorname{ext}(K)$ is weak*-dense in ext $(K)$, and the weak*closed convex hull of $A \cap \operatorname{ext}(K)$, is $K$.

Proof. It is only enough to apply Theorem 4.3 to the linear space $H$ with the norm and weak ${ }^{*}$ topologies in places of $\mathscr{T}_{1}$ and $\mathscr{T}_{2}$ respectively. Conditions (i) and (ii) are trivially satisfied, and Theorem 4.7 states that condition (ii) is fulfilled.

A special case of Theorem 4.8, where $E^{*}$ is separable, was proved in [18, Theorem 3.2]. This theorem had some interesting consequences about the existence of extreme points and denting points [18, Corollary 3.4 and Theorem 3.5]. By repeating exactly the same simple arguments, we obtain from Theorem 4.8 the following corollary.

CoRollary 4.9. Let $E$ be a normed linear space such that $E^{*}$ is weak-compactly generated. Then

(a) each norm-closed, convex, bounded subset of $E^{*}$ is the normclosed convex hull of its extreme points, and

(b) each norm-closed, convex, bounded subset of $E^{*}$ is contained in the weak*-closed convex hull of its denting points. ${ }^{2}$

REMARK 4.10. The proof of (a) requires [16, Lemma 1] which was proved by Lindenstrauss using a deep theorem of Bishop and Phelps [3]. We wish to point out that there is a simple but elegant alternative argument by Bourgin [5] (see [19, Lemma 1]).

Conclusion (a) of the preceding theorem answers Problem 16 of Lindenstrauss [17] affirmatively. ${ }^{3}$ Previously John and Zizler obtained the same conclusion with the additional hypothesis that $E$ be a weakcompactly generated Banach space [12, Corollary 3]. Their proof

${ }^{2}$ In a forthcoming paper entitled "Dentability and extreme points in Banach spaces", R. R. Phelps derives from Theorem 4.8 a conclusion stronger than Corollary 4.9 .

${ }^{3}$ This problem was also solved recently by Lindenstrauss by a completely different method. 
consists of a modification of Troyanski's method.

There is another class of dual spaces where the conclusions of Theorem 4.8 and Corollary 4.9 are valid. Let us say that the norm of the dual $E^{*}$ of a normed linear space $E$ satisfies condition ( $\star \star \star$ ) if a net $\left\{f_{\gamma}\right\}$ in $E^{*}$ converges to $f$ in norm whenever $f_{\gamma} \rightarrow f$ relative to the weak* topology and $\left\|f_{r}\right\| \rightarrow\|f\|$. This is equivalent to saying that, on the unit sphere $\{f:\|f\|=1\}$, the weak* topology coincides with the norm topology. For instance, the norm of $l_{1}(S)=\left(c_{0}(S)\right)^{*}$ satisfies condition $(\star \star)$. The next proposition is not directly related to the main theme of the present paper, but it is an easy consequence of Theorem 4.3.

Proposition 4.11. Let $E$ be a normed linear space such that the norm of $E^{*}$ satisfies ( $\left.\star \star \star\right)$, let $K$ be a weak*-compact convex subset of $E^{*}$, and let $A$ be the set of all points of continuity of the identity map: $\left(K\right.$, weak $\left.^{*}\right) \rightarrow(K$, norm $)$. Then $A \cap \operatorname{ext}(K)$ is weak*-dense in ext $(K)$. Consequently conclusions (a) and (b) of Corollary 4.9 are also valid for $E^{*}$.

Proof. In view of Theorem 4.3, it is sufficient to establish the following: If $D$ is a weak*-compact subset of $E^{*}$ then the identity map $\left(D\right.$, weak $\left.{ }^{*}\right) \rightarrow(D$, norm $)$ is continuous at points of a weak*-dense subset of $D$. Since the norm function $f \mapsto\|f\|$ is lower weak*semicontinuous on $E^{*}$ and since $(D$, weak $)$ is Baire, there is a dense $G_{\sigma^{-}}$-set $B$ in $\left(D\right.$, weak $\left.{ }^{*}\right)$ such that the norm function restricted to $D$ is weak*-continuous at points of $B$. Suppose that $\left\{f_{r}\right\}$ is a net in $D$ such that $f_{r} \rightarrow f \in B$ relative to the weak* topology. Then $\left\|f_{r}\right\| \rightarrow$ $\|f\|$, and it follows from property ( $\star \star)$ that $f_{r} \rightarrow f$ in the norm topology, i.e., the identity map: $\left(D\right.$, weak $\left.{ }^{*}\right) \rightarrow(D$, norm $)$ is continuous at points of $B$. This concludes the proof.

Proposition 4.11 was independently discovered by J. Lindenstrauss and by us in 1967. The proof of Lindenstrauss is outlined in a letter to Asplund, and it is based on a modification of [15, Theorem 1]. Asplund gave a different proof of the fact that $l_{1}(S)$ has the "KreinMilman property" (i.e., the conclusion (a) of Corollary 4.9) in [2]. When the set $S$ is uncountable, the space $l_{1}(S)$ is not weak-compactly generated.

\section{REFERENCES}

1. D. Amir and J. Lindenstrauss, The structure of weakly compact sets in Banach spaces, Ann. of Math., 88 (1968), 35-46.

2. E. Asplund, Boundedly Krein-compact spaces, Proceedings of the Functional Analysis week, March 3-7, 1969, Math. Inst., Aarhus Univ.

3. E. Bishop and R. R. Phelps, The support functionals of a convex set, Proc. Symposia 
in Pure Math. VII (Convexity), Amer. Math. Soc., (1963), 27-37.

4. N. Bourbaki, Topologie générale, Chapitre 9, Deuxième Édition, Actualitiés Sci., Ind. No. 1045, Hermann, Paris, 1958.

5. R. D. Bourgin, Barycenters of measures on certain noncompact convex sets, Thesis, University of Washington, 1969.

6. H. H. Corson and I. Glicksberg, Compactness in Hom $(G, H)$, Canada J. Math., 22 (1970), 164-170.

7. R. Ellis, Locally compact transformation groups, Duke Math. J., 24 (1957), 119125.

8. R. Engelking, Outline of General Topology, North-Holland Publishing Co., Amsterdam, 1968.

9. M. K. Fort, Jr., Category theorems, Fund. Math., 42 (1955), 276-288.

10. Z. Frolik, Generalizations of the Gs-property of complete metric spaces, Czech. Math. J., 10 (1960), 359-379.

11. - Baire spaces and some generalizations of complete metric spaces, Czech. Math. J., 11 (1961), 237-238.

12. K. John and V. Zizler, A renorming of dual spaces. Israel Math. J., 12 (1972), 331-336.

13. J. L. Kelley, General Topology, D. Van Nostrand Co. Inc., 1955.

14. J. L. Kelley, I. Namioka et al., Linear Topological Spaces, D. Van Nostrand Co., Inc., 1963.

15. J. Lindenstrauss, On operators which attain their norm, Israel J. Math., 1 (1963), $139-148$.

16. - On extreme points in $l_{1}$, Israel J. Math., 4 (1966), 59-61.

17. - Weakly compact sets, their topological properties and Banach spaces they generate, Proc. Symp. Infinite Dim. Topology 1967, Annals of Math. Studies, Princeton Univ. Press, 1972.

18. I. Namioka, Neighborhoods of extreme points, Israel J. Math., 5 (1967), 145-152.

19. N. T. Peck, Support points in locally convex space, Duke Math. J., 38 (1971), 271-278.

20. M. A. Rieffel, Dentable subsets of Banach spaces with applications to a RadonNikodym theorem, Functional Analysis (Proc. Conf., Irvine, Calif., 1966), pp. 71-77. Thompson Book Co., Washington, D. C., 1967.

21. S. L. Troyanski, On locally uniformly convex and differentiable norms in certain nonsepparable Banach spaces, Studia Math., 37 (1971), 173-180.

Received October 17, 1973. This research was supported, in part, by NSF-GP-29107.

UNIVERSITY OF WASHINGTON 



\section{PACIFIC JOURNAL OF MATHEMATICS}

\section{EDITORS}

RICHARD ARENS (Managing Editor)

University of California

Los Angeles, California 90024
J. DUGUNDJI*

Department of Mathematics

University of Southern California

Los Angeles, California 90007

D. Gilbarg and J. Milgram

Stanford University

Stanford, California 94305
University of Washington

Seattle, Washington 98105

ASSOCIATE EDITORS
E. F. BECKENBACH
B. H. NeumanN
F. WoLF
K. YosHIDA

\section{SUPPORTING INSTITUTIONS}

\author{
UNIVERSITY OF BRITISH COLUMBIA \\ CALIFORNIA INSTITUTE OF TECHNOLOGY \\ UNIVERSITY OF CALIFORNIA \\ MONTANA STATE UNIVERSITY \\ UNIVERSITY OF NEVADA \\ NEW MEXICO STATE UNIVERSITY \\ OREGON STATE UNIVERSITY \\ UNIVERSITY OF OREGON \\ OSAKA UNIVERSITY
}

\author{
UNIVERSITY OF SOUTHERN CALIFORNIA \\ STANFORD UNIVERSITY \\ UNIVERSITY OF TOKYO \\ UNIVERSITY OF UTAH \\ WASHINGTON STATE UNIVERSITY \\ UNIVERSITY OF WASHINGTON
* * * *
AMERICAN MATHEMATICAL SOCIETY \\ NAVAL WEAPONS CENTER
}

The Supporting Institutions listed above contribute to the cost of publication of this Journal, but they are not owners or publishers and have no responsibility for its content or policies.

Mathematical papers intended for publication in the Pacific Journal of Mathematics should be in typed form or offset-reproduced, (not dittoed), double spaced with large margins. Underline Greek letters in red, German in green, and script in blue. The first paragraph or two must be capable of being used separately as a synopsis of the entire paper. Items of the bibliography should not be cited there unless absolutely necessary, in which case they must be identified by author and Journal, rather than by item number. Manuscripts, in duplicate if possible, may be sent to any one of the four editors. Please classify according to the scheme of Math. Rev. Index to Vol. 39. All other communications to the editors should be addressed to the managing editor, or Elaine Barth, University of California, Los Angeles, California, 90024.

100 reprints are provided free for each article, only if page charges have been substantially paid. Additional copies may be obtained at cost in multiples of 50 .

The Pacific of Journal Mathematics is issued monthly as of January 1966. Regular subscription rate: $\$ 72.00$ a year (6 Vols., 12 issues). Special rate: $\$ 36.00$ a year to individual members of supporting institutions.

Subscriptions, orders for back numbers, and changes of address should be sent to Pacific Journal of Mathematics, 103 Highland Boulevard, Berkeley, California, 94708.

PUBLISHED BY PACIFIC JOURNAL OF MATHEMATICS, A NON-PROFIT CORPORATION

Printed at Kokusai Bunken Insatsusha (International Academic Printing Co., Ltd.), 270, 3-chome Totsuka-cho, Shinjuku-ku, Tokyo 160, Japan.

* C. R. DePrima California Institute of Technology, Pasadena, CA 91109, will replace J. Dugundji until August 1974.

Copyright (C) 1973 by Pacific Journal of Mathematics

Manufactured and first issued in Japan 


\section{Pacific Journal of Mathematics}

\section{Vol. 51, No. $2 \quad$ December, 1974}

Robert F. V. Anderson, Laplace transform methods in multivariate spectral theory .................................................. 339

William George Bade, Two properties of the Sorgenfrey plane . . . . . . . . . . . . 349

John Robert Baxter and Rafael Van Severen Chacon, Functionals on continuous

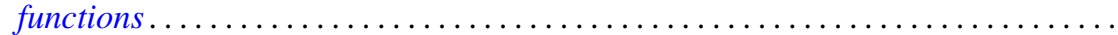

Phillip Wayne Bean, Helly and Radon-type theorems in interval convexity

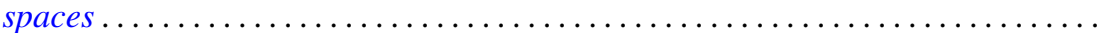

James Robert Boone, On k-quotient mappings $\ldots \ldots \ldots \ldots \ldots \ldots \ldots \ldots \ldots$

Ronald P. Brown, Extended prime spots and quadratic forms . . . . . . . . . . . .

William Hugh Cornish, Crawley's completion of a conditionally upper continuous lattice .............................................

Robert S. Cunningham, On finite left localizations ...................

Robert Jay Daverman, Approximating polyhedra in codimension one spheres

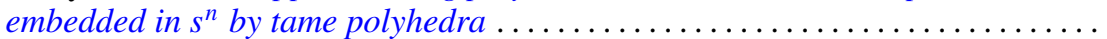

Burton I. Fein, Minimal splitting fields for group representations . . . . . . . . . . . .

Peter Fletcher and Robert Allen McCoy, Conditions under which a connected

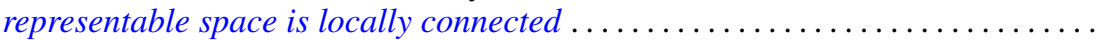

Jonathan Samuel Golan, Topologies on the torsion-theoretic spectrum of a noncommutative ring...

Manfred Gordon and Edward Martin Wilkinson, Determinants of Petrie matrices.

Alfred Peter Hallstrom, A counterexample to a conjecture on an integral condition for determining peak points (counterexample concerning peak points)........

E. R. Heal and Michael Windham, Finitely generated $F$-algebras with applications to Stein manifolds.

Denton Elwood Hewgill, On the eigenvalues of a second order elliptic operator in an unbounded domain ............................

Charles Royal Johnson, The Hadamard product of $A$ and $A^{*}$.

Darrell Conley Kent and Gary Douglas Richardson, Regular completions of Cauchy spaces.

Alan Greenwell Law and Ann L. McKerracher, Sharpened polynomial approximation

Bruce Stephen Lund, Subalgebras of finite codimension in the algebra of analytic functions on a Riemann surface. .

Robert Wilmer Miller, TTF classes and quasi-generators . .

Roberta Mura and Akbar H. Rhemtulla, Solvable groups in which every maximal partial order is isolated ....

Isaac Namioka, Separate continuity and joint continuity...

Alan Saleski, Entropy of self-homeomorphisms of statistical pseudo-metric

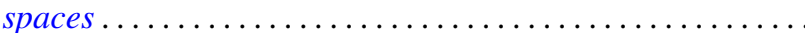

H. A. Seid, Cyclic multiplication operators on $L_{p}$-spaces .....

H. B. Skerry, On matrix maps of entire sequences ............

John Brendan Sullivan, A proof of the finite generation of invariants of a normal

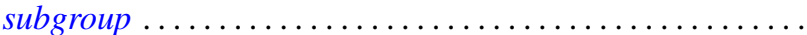

John Griggs Thompson, Nonsolvable finite groups all of whose local subgroups are

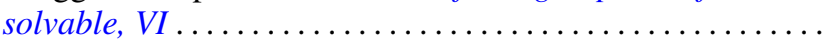

